\title{
The oscillation effect at near-threshold intensities during sequential alternation of unilateral amygdaloid stimulation
}

\author{
JOHN GAITO \\ York University, Downsview, Ontario, Canada MSJ1P3
}

\begin{abstract}
Previous research had indicated that an oscillation effect resulted during sequential alternation of unilateral amygdaloid stimulation with consistently low latency values for one side and consistently high values for the contralateral one. The effect of intensities approximately 15 microA above threshold was investigated in this study. The oscillation tendency occurred at these near-threshold intensities both for rats which previously had shown oscillation patterns at different intensities and for those which were being stimulated for the first time.
\end{abstract}

The "kindling effect" has been investigated in a number of laboratories as a model of learning, a model of epilepsy, or as an example of behavioral change of interest in its own right (e.g., Gaito \& Gaito, 1974; Goddard, McIntyre, \& Leech, 1969). This event involves a change from normal exploration (Stage 1: NE), to behavioral automatisms (Stage 2: BA, chewing, eye closure on ipsilateral side, salivation), and finally to clonic convulsions (Stage 3: CC) in response to electrical stimulation of a specific brain site (e.g., amygdala). Stage 3 behavior involves the rat standing on its hind paws, and bilateral convulsions of the forelimbs ensue. Behavioral, chemical, electrophysiological, and neurological aspects of this effect have been investigated by many researchers (Gaito, 1976a).

If one amygdala (primary site) is stimulated with 50-microA intensity for $5 \mathrm{sec}$ to a criterion (e.g., six clonic convulsions-CC), using $24 \mathrm{~h}$ as the intertrial interval, and then the contralateral amygdala (secondary site) is stimulated, the criterion for the latter is reached within a fewer number of trials, but increased latency to convulsion results. Thus, both positive and negative transfer occur (Goddard et al., 1969; McIntyre \& Goddard, 1973). If the animal is stimulated again at the primary site, it does not convulse on the first trial. A few trials are required to reach the $\mathrm{CC}$ stage, thus indicating negative transfer.

In recent research in our laboratory with 3 trials/day and stimulation at an intensity of 100 microA for $30 \mathrm{sec}$ using a sequence of alternating unilateral stimulations of the amygdala, usually for 10 alternation phases, an "oscillation effect" was observed (Gaito, 1976b). This oscillation usually involved low values consistently for the amygdala first stimulated (primary site) and high values consistently for the contralateral amygdala (secondary site). The effect was most prominent in latency data (number of seconds from onset of stimulation to onset of convulsion).

A number of variables which might affect this oscil- lation tendency have been investigated in our laboratory. In latency data bilateral stimulation prior to, or after, the development of oscillation had no disruptive effect on the oscillation tendency (Gaito, 1976c). The tendency persisted with 1, 3, or 6 trials/day (Gaito, 1976d) and 1,6 , or 12 convulsions/phase (Gaito, 1976e). Oscillation was as prominent in old rats $(420-475$ days of age) as in younger ones (135-200 days) (Gaito \& Nobrega, 1977). Rest intervals of 1,3 , and 6 months interspersed between two sets of 10 sequential alternation phases had no deleterious effect (Gaito, 1976f). The tendency was unaffected by intensities of 100 and 280 microA, but 560-microA intensity had a slight disruptive effect (Gaito, 1977). Thus, it appears that the oscillation effect is an extremely robust event for latency data.

In the criterion measure (number of trials to a specific number of convulsions, usually six), there were few oscillators. Each of the above variables disrupted partially, or completely, this weak oscillation tendency. The duration of convulsion measure was not analyzed in these experiments because few oscillators were detected in the first study.

There are two types of oscillation which occur consistently in our research. Primary oscillation involves low values for the primary site and high values for the secondary site for at least 8 of 10 phases. Secondary oscillation is the opposite pattern. Table 1 shows data for 139 rats stimulated over 10 alternating phases in the previous research.

Although the oscillation effect appears to be an authentic response of the amygdala to sequential alternation of unilateral stimulation, there are a number of possible artifactual bases for these results. These are: chance results, one electrode is misplaced, and constant intensity for both sides favors one side because of differential thresholds for convulsions.

The idea that the oscillation results are due to chance must be rejected. With our procedure of alternating 
Table 1

Frequency of Different Types of Behavior With Each Measure

\begin{tabular}{lrrrc}
\hline & PO & SO & NO & Total \\
\hline Latency & 59 & 44 & 36 & 139 \\
Criterion & 28 & 23 & 88 & 139 \\
Duration & 37 & 25 & 77 & 139 \\
Total & 124 & 92 & 201 & 417 \\
\hline
\end{tabular}

Note-PO $=$ low values in primary site and high values in secondary site for at least 8 of 10 phases; SO =low values in secondary site and high values in primary site for at least 8 of 10 phases; $N O=$ lack of systematic pattern.

stimulations over 10 phases and our requirement that a rat must show a consistent "low-high" or "high-low" pattern for at least 8 of the 10 phases to be designated as an "oscillator," the probability that these apparent systematic patterns of oscillation are actually random ones is $.17 .^{1}$ The number of oscillators indicated in Table 1 with 139 rats involved in previous experiments is significantly different from the expected value of 23.6 in each of the three measures. The greatest number of oscillators occurs in latency data. Thus, this measure appears to be the most sensitive indicator of oscillation, an observation which was obvious from the first study.

The misplaced electrode explanation appears to be a reasonable one. However, it does not seem to be a major contributor, for a number of reasons cited previously (Gaito, 1976b, 1976c, 1977). This possibility will be evaluated by future experiments in which one electrode will be placed in the amygdala and the other in nearby tissue for some rats.

The previous experiments were conducted with the same intensity of stimulation for both amygdalae. However, it is possible that slight differences in the placement of electrodes in the amygdalae could result in differential intensities required to elicit the clonic convulsion. Thus, the same intensities in both amygdalae might result in the $\mathrm{CC}$ condition being reached for one amygdala in fewer trials than for the other one, and this aspect might be the basis for the oscillation effect in latency and criterion measures. The present study was concerned with an evaluation of this possibility. Intensities just above threshold were used with each amygdala. This part of the study was concerned with two aspects: (1) the effect of stimulation just above threshold intensities over 10 phases of sequential alternation after rats had developed the oscillation tendency (Experiment 1), and (2) the effect of suprathreshold stimulation during the development of kindling over 10 phases of sequential alternation (Experiment 2).

A secondary purpose of this study was to describe in more detail the "abortive CCs" ("incomplete CCs" which terminate in less than $5 \mathrm{sec})$ that have been observed in previous research (Gaito, 1976c, 1976d, 1976e, 1977; Gaito \& Nobrega, 1977). These "abortive CCs" have been found to be more prominent during stimulation of the "high" than of the "low" side. This description occurred in Experiment 1.

\section{METHODS}

For Experiment 1, 11 male Wistar rats were chosen from previous research (between 200 and 300 days of age); eight of the animals had shown oscillation tendencies in latency data and had presented a number of abortive CCs. The brain coordinates used for implantation of bipolar electrodes into the amygdalae were the same as in many experiments in our laboratory: $.5 \mathrm{~mm}$ posterior to bregma, $4.5 \mathrm{~mm}$ from midline, $8.5 \mathrm{~mm}$ from skull (Gaito \& Gaito, 1974; Gaito, 1976b).

The 11 rats were stimulated with a $60-\mathrm{Hz}$ sine wave over 10 phases of alternating unilateral sites for $30 \mathrm{sec}$ with a Lafayette stimulator until six convulsions occurred for each phase. Stimulation began on the "high" or "low" side based on a random process. On the first trial of Phases 1 and 2, the intensity was slowly increased until a definite response to the current was indicated. Then the current was increased approximately 15 microA beyond that point. The first CC trial in each of these phases was not used in the data analyses, because varying amounts of time were required to find the near-threshold intensities for each side. The intensities used were between 84 and 196 microA for most rats; one required 280 microA on one side and two others had 420 microA on one side.

In Experiment 2, each of 12 male Wistar rats (approximately 150 days of age) were implanted bilaterally with electrodes in the amygdalae. Stimulation of 100 -microA intensity for 30 -sec duration began 7 days after surgery. With some rats the intensity had to be increased to achieve convulsion during Phases 1 and 2 . Threshold values were determined during Phases 3 and 4 and stimulation intensities were set approximately 15 micro $A$ above threshold for each rat. The intensities used were approximately the same as in Experiment 1.

In the first study (Gaito, 1976b), histological analyses were completed on 13 of the 19 rats involved and indicated that the electrode tips were in both amygdalae in 11 of the 13 rats. However, oscillation resulted whether electrodes were in the amygdalae or in nearby tissue; therefore, histological analyses were not conducted in later experiments, including the present ones.

\section{RESULTS}

The 11 rats in Experiment 1 consisted of six primary oscillators (low values in primary site, high values in secondary site), two secondary oscillators (high values

Table 2

Types of Oscillation in the Two Series of 10 Phases

\begin{tabular}{cccccc}
\hline $\begin{array}{c}\text { Rat } \\
\text { Number }\end{array}$ & $\begin{array}{c}\text { Previous } \\
\text { Research }\end{array}$ & $\begin{array}{c}\text { Present } \\
\text { Experiment }\end{array}$ & $\begin{array}{c}\text { Rat } \\
\text { Number }\end{array}$ & $\begin{array}{c}\text { Previous } \\
\text { Research }\end{array}$ & $\begin{array}{c}\text { Present } \\
\text { Experiment }\end{array}$ \\
\hline 1 & PO & PO & 7 & SO & NO \\
2 & PO & SO & 8 & PO & PO \\
3 & PO & PO & 9 & PO & PO \\
4 & NO & NO & $10^{*}$ & SO & SO \\
5 & NO & PO & $11^{*}$ & NO & PO \\
6 & PO & NO & & & \\
\hline
\end{tabular}

Note $-P O=$ primary oscillation, $S O=$ secondary oscillation, $N O=$ nonoscillation.

*Lost electrode after four phases. 
Table 3

Typical Oscillation Responses for Latency (L) and Criterion (C) Measures

\begin{tabular}{|c|c|c|c|c|c|c|c|c|c|c|c|c|c|c|c|}
\hline \multirow{2}{*}{$\begin{array}{c}\text { Rat } \\
\text { Number }\end{array}$} & & \multicolumn{4}{|c|}{$\begin{array}{c}\text { Last Phases of Previous } \\
\text { Research }\end{array}$} & \multicolumn{10}{|c|}{ Phases of Present Experiment } \\
\hline & & 7 & 8 & 9 & 10 & 1 & 2 & 3 & 4 & 5 & 6 & 7 & 8 & 9 & 10 \\
\hline 1 & $\begin{array}{l}\mathrm{L} \\
\mathrm{C}\end{array}$ & $\begin{array}{l}\mathrm{L} \\
1.3 \\
6\end{array}$ & $\begin{array}{l}\mathrm{R} \\
7.3 \\
6\end{array}$ & $\begin{array}{l}\mathrm{L} \\
1.0 \\
6\end{array}$ & $\begin{array}{l}\mathrm{R} \\
5.7 \\
6\end{array}$ & $\begin{array}{l}\mathrm{R} \\
8.4 \\
7\end{array}$ & $\begin{array}{l}\mathrm{L} \\
4.2 \\
6\end{array}$ & $\begin{array}{c}\mathbf{R} \\
10.3 \\
8\end{array}$ & $\begin{array}{l}\mathrm{L} \\
4.8 \\
6\end{array}$ & $\begin{array}{l}\mathrm{R} \\
9.0 \\
6\end{array}$ & $\begin{array}{l}\mathrm{L} \\
4.3 \\
6\end{array}$ & $\begin{array}{l}\mathrm{R} \\
8.7 \\
6\end{array}$ & $\begin{array}{l}\mathrm{L} \\
5.7 \\
6\end{array}$ & $\begin{array}{l}\mathrm{R} \\
6.2 \\
6\end{array}$ & $\begin{array}{l}\mathrm{L} \\
6.0 \\
6\end{array}$ \\
\hline 2 & $\begin{array}{l}\mathrm{L} \\
\mathrm{C}\end{array}$ & $\begin{array}{l}\mathrm{R} \\
6.0 \\
6\end{array}$ & $\begin{array}{c}\mathrm{L} \\
12.5 \\
9\end{array}$ & $\begin{array}{l}\mathrm{R} \\
2.8 \\
6\end{array}$ & $\begin{array}{l}\mathrm{L} \\
4.8 \\
6\end{array}$ & $\begin{array}{l}\mathrm{R} \\
8.6 \\
6\end{array}$ & $\begin{array}{c}\mathrm{L} \\
11.6 \\
6\end{array}$ & $\begin{array}{c}\mathrm{R} \\
10.0 \\
6\end{array}$ & $\begin{array}{l}\mathrm{L} \\
9.2 \\
6\end{array}$ & $\begin{array}{c}\mathrm{R} \\
11.7 \\
6\end{array}$ & $\begin{array}{l}\text { L } \\
8.7 \\
7\end{array}$ & $\begin{array}{l}\mathrm{R} \\
9.3 \\
6\end{array}$ & $\begin{array}{l}\mathrm{L} \\
5.3 \\
6\end{array}$ & $\begin{array}{c}\mathrm{R} \\
12.7 \\
6\end{array}$ & $\begin{array}{l}\text { L } \\
5.7 \\
6\end{array}$ \\
\hline 4 & $\begin{array}{l}\mathrm{L} \\
\mathrm{C}\end{array}$ & $\begin{array}{l}\mathrm{R} \\
2.3 \\
6\end{array}$ & $\begin{array}{l}\mathrm{L} \\
1.7 \\
6\end{array}$ & $\begin{array}{l}\mathrm{R} \\
3.4 \\
6\end{array}$ & $\begin{array}{l}\mathrm{L} \\
1.7 \\
6\end{array}$ & $\begin{array}{l}\mathrm{R} \\
7.4 \\
6\end{array}$ & $\begin{array}{l}\mathrm{L} \\
7.0 \\
6\end{array}$ & $\begin{array}{l}\mathrm{R} \\
6.5 \\
6\end{array}$ & $\begin{array}{l}\mathrm{L} \\
5.7 \\
6\end{array}$ & $\begin{array}{l}\mathrm{R} \\
4.8 \\
6\end{array}$ & $\begin{array}{l}L \\
7.0 \\
6\end{array}$ & $\begin{array}{l}\mathrm{R} \\
5.7 \\
6\end{array}$ & $\begin{array}{l}L \\
L \\
8.8 \\
6\end{array}$ & $\begin{array}{l}\mathrm{R} \\
4.2 \\
6\end{array}$ & $\begin{array}{l}\text { L } \\
6.7 \\
6\end{array}$ \\
\hline
\end{tabular}

Note-L = mean of six trials in each phase, in seconds; $C=$ number of trials to six CCs. L (left) and $R$ (right) under phase numbers refer to the side of the brain being stimulated.

in primary site, low values in secondary site), and three nonoscillators. In previous research primary oscillation had occurred most frequently. Two rats lost electrodes after four phases and had to be discarded (one had been a nonoscillator; the other was a secondary oscillator).

Six of the nine rats which completed the 10 phases showed oscillation in the latency measure, but none oscillated in criterion data. Five had the same oscillation pattern in latency data as in the previous research, two changed to nonoscillation, one nonoscillator changed to SO, and a second nonoscillator continued the NO pattern. Change of oscillation pattern is an infrequent event (Gaito \& Nobrega, 1977). One of the two rats which lost the electrode also showed the same oscillation pattern during the four phases as it had during the earlier research. The other rat, which had been a nonoscillator, showed a primary oscillation pattern. Thus, 8 of the 11 rats oscillated.

Typical data for the last 4 phases of the earlier experiments and 10 phases of the present one are given in Table 3 for three rats. The first one showed primary oscillation for both sets in latency data. A second reversed from primary to secondary oscillation after Phase 2 for the latency measure. The third was a nonoscillator in both cases for latency data.

In Experiment 2, 8 of the 12 rats showed oscillation behavior in criterion and in latency data, with primary oscillation predominating in both cases (Table 4). With the probability of being designated as an oscillator equal to .17 , the expected number of oscillators with 12 rats is 2.04 or 2 . Using the binomial distribution, the probability that eight or more rats will oscillate is .00019 (or .00038 if both ends of the distribution are considered). Thus, the observed number of oscillators in both latency and criterion measures are significantly different from chance (using an $\alpha$ level of .01 for rejection of null hypotheses).

The data indicating abortive $\mathrm{CCs}$ for the nine rats which completed the 10 phases in Experiment 1 are shown in Table 5. The mean number of these responses was 11.6 for the high side and 6.2 for the low side. Six rats showed a greater number of these responses on the high side, for two this pattern was reversed, and one rat had an equal number of responses for each side. Three rats showed a large number of abortive CCs on the low side (Rats 3, 4, and 6). Rats 4 and 6 were nonoscillators in the 10 phases, which might explain the large number of abortive CCs on both sides. However, Rat 3 had been a primary oscillator in the previous research and continued this pattern for the entire 10 phases of the present experiment.

Table 4

Pattern of Behavior of Rats in Experiment 2

\begin{tabular}{cccccc}
\hline $\begin{array}{c}\text { Rat } \\
\text { Number }\end{array}$ & Criterion & Latency & $\begin{array}{c}\text { Rat } \\
\text { Number }\end{array}$ & Criterion & Latency \\
\hline 1 & PO & PO & 7 & PO & PO \\
2 & PO & PO & 8 & NO & NO \\
3 & NO & NO & 9 & NO & SO \\
4 & SO & PO & 10 & PO & SO \\
5 & SO & NO & 11 & NO & PO \\
6 & SO & NO & 12 & PO & PO \\
& & & & 5 POs & 6 POs \\
& & & & 3 SOs & 2 SOs \\
& & & & 4 NOs & 4 NOs \\
\hline
\end{tabular}

Table 5

Number of Trials of Abortive CCs for High and Low Sides in Experiment 1

\begin{tabular}{crrcrc}
\hline $\begin{array}{c}\text { Rat } \\
\text { Number }\end{array}$ & High & Low & $\begin{array}{c}\text { Rat } \\
\text { Number }\end{array}$ & High & Low \\
\hline 1 & 7 & 0 & 6 & 8 & 10 \\
2 & 25 & 3 & 7 & 9 & 2 \\
3 & 2 & 12 & 8 & 11 & 5 \\
4 & 22 & 15 & 9 & 18 & 7 \\
5 & 2 & 2 & & & \\
Mean & & & & 11.6 & 6.2 \\
\hline
\end{tabular}




\section{DISCUSSION}

The results of the previous experiments indicated the occurrence of the oscillation effect in the latency measure, and occasionally in criterion data, when the intensity of stimulation was constant for both amygdala. The results of Experiments 1 and 2 show that oscillation still persists in latency when intensities are just above threshold values. However, not a single rat oscillated in the criterion measure in Experiment 1, but 8 of 12 showed an oscillation pattern in Experiment 2. Thus, these results are further evidence of the occurrence of the oscillation effect, especially in latency data, and indicate that this effect is not based on differential effective intensities in the two hemispheres.

The presence of abortive CCs during stimulation of the high side was suggested as evidence of the authenticity of the oscillation effect (Gaito, 1976c, 1976d, 1976e, 1977; Gaito \& Nobrega, 1977). These events were interpreted as evidence of an inhibitory process which develops during unilateral stimulation. The results of Experiment 1 were consistent with this interpretation. Abortive CCs tended to be more frequent on the high side; these results seem to suggest that some active process tends to impede the occurrence of the convulsion.

\section{REFERENCES}

Garto, J. The kindling effect as a model of epilepsy. Psychological Bulletin, 1976, 83, 1097-1109. (a)

GAITo, J. An oscillation effect during sequential alternations of unilateral amygdaloid stimulations within the kindling paradigm. Physiological Psychology, 1976, 4, 303-306. (b)

GaITo, J. The effect of bilateral stimulation during sequential alternation of unilateral amygdaloid stimulation. Bulletin of the Psychonomic Society, 1976, 4, 355-357. (c)

GaITo, J. The effect of number of trials per day during sequential alternation of unilateral amygdaloid stimulation. Bulletin of the Psychonomic Society, 1976, 4, 403-404. (d)

GaIto, J. The effect of number of convulsions per phase on the oscillation tendency. Bulletin of the Psychonomic Society, 1976, 8, 392-394. (e)

GaIto, J. The effect of varying rest intervals following the development of oscillation during unilateral amygdaloid stimulation. Bulletin of the Psychonomic Society, 1976, 8, 457-458. (f)

Garto, J. The effect of intensity during sequential alternation of unilateral amygdaloid stimulation. Bulletin of the Psychonomic Society, 1977, 9, 64-66.

GaIto, J., \& GaIto, S. T. Interanimal negative transfer of the kindling effect. Physiological Psychology, 1974, 2, 379-382.

Gaito, J., \& NobregA, J. The oscillation effect during sequential alternation of amygdaloid stimulation with aged rats. Bulletin of the Psychonomic Society, 1977, 9, 151-154.

Goddard, G. V., McIntyre, D. C., \& LeECh, C. K. A permanent change in brain function resulting from daily electrical stimulation. Experimental Neurology, 1969, 25, 295-330.

HoEl, P. G. Introduction to mathematical statistics. New York: Wiley, 1954.

MCINTYRE, D. C., \& GoddARD, G. V. Transfer, interference and spontaneous recovery of convulsions kindled from the rat amygdala. Electroencephalography and Clinical Neurophysiology, 1973, 35, 533-543.

\section{NOTE}

1. The hypothesis that the oscillation patterns are random ones can be assessed by the one-sample runs test (Hoel, 1954). Using the requirement of 8 or 10 phases of oscillation out of 10 phases would provide 8,9 , or 10 runs. The $p$ of 8,9 , and 10 runs is $.1270, .0317$, and .0079 , respectively; the $p$ of 8,9 , or 10 runs is the sum of these ps, .1667 or .17 . Thus, the $p$ that these apparent systematic patterns are random ones would be .17 .

(Received for publication April 4, 1977.) 